\title{
Malignant Transformation of a Mature Ovarian Teratoma: a Case Report and Review of the Literature
}

\author{
Ru-bing Huª, Yun-xiao Zhou ${ }^{b^{*}}$, Xiao-qing Jin', Cheng-dong Changd, Hao Wanga, Bao-mei Shi', Jin \\ Shi $^{\text {b }}$ \\ ${ }^{a}$ Department of Gynecology, Yiwu Central Hospital. \\ ${ }^{b}$ Department of Gynecology, The First Affiliated Hospital, Zhejiang University. \\ 'Department of Pathology, Yiwu Central Hospital. \\ ${ }^{d}$ Department of Pathology, The First Affiliated Hospital, Zhejiang University.
}

\begin{abstract}
A mature ovarian teratoma is a common benign germ cell tumor of the ovary in women that rarely causes a malignant transformation. However, a malignant transformation can occur in the ectodermal, mesodermal, and endodermal tissues of the tumor. A squamous cell carcinoma is the most common and an adenocarcinoma is the second most common malignant tumor derived from a cystic teratoma. Due to the rarity of malignant transformations in mature teratomas and the lack of clinical data, the clinical pathological features, malignant transformation mechanism, therapeutic methods, and prognosis-relevant factors are unclear. Currently, there is no specific diagnostic method, and it is a difficult lesion to diagnose before performing surgery. In order to improve clinicians' understanding of a malignant-transformed mature teratoma and identification of malignant-transformed teratomas at early stages, we have reported a case of a mature teratoma with the malignant transformation of a sebaceous gland carcinoma in the Yiwu Central Hospital in Zhejiang, China on May 31, 2018. A review of the literature is also presented to summarize the clinical symptoms, pathogenesis, diagnosis, and treatment of malignant-transformed mature teratomas.

Keywords: mature teratoma; malignant transformation; sebaceous gland carcinoma
\end{abstract}

\section{INTRODUCTION}

Ovarian teratomas derive from germ cells with totipotent differentiation potential, and they include the ectoderm, mesoderm, and endoderm, which can be divided into mature and immature teratomas. A mature teratoma is the most common benign germ cell tumor of the ovary, and it can be further divided into solid and cystic types, the cystic type of which is very common. It mainly occurs in women of childbearing age, with a median age of 30 years old ${ }^{[1]}$. One part of a mature teratoma can cause a malignant transformation. However, the malignant transformation rate is low, accounting for $1 \%-3 \%$ of all teratomas, and these are usually found in pre and postmenopausal women ${ }^{[1,2]}$. Here, we have described the case of a mature teratoma with a malignant transformation along with its clinicopathological features.

*Corresponding author: Yun-xiao Zhou

Mailing address: Department of Gynecology, The First Affiliated Hospital, Zhejiang University. No. 79, Qingchun Road, Shangcheng District, Hangzhou, Zhejiang.

E-mail: zhouyx2017@zju.edu.cn

Received: 20 July 2018 Accepted: 25 September 2018

\section{CASE REPORT}

A 55-year-old woman who had been going through menopause for 4 years was admitted to the hospital due to pelvic lumps found in her physical examination. In April of 2018, a B-mode ultrasound showed a left adnexal mass in this patient, which was considered to be a teratoma. Computed tomography (CT) scanning of the pelvic cavity showed that the left adnexal area contained fat and a solid cystic mass, which were considered to be part of the teratoma. After this patient was admitted, she underwent additional relevant examinations in order to clarify the diagnosis. Her serum cancer antigen (CA)125 level was $48.9 \mathrm{U} / \mathrm{ml}$, her CA199, carcinoembryonic antigen (CEA), alpha-fetoprotein (AFP), and squamous cell carcinoma (SCC) levels were within the normal ranges, and no obvious abnormalities were found in the remaining examination.

On May 31, a laparoscopic bilateral adnexectomy was conducted under general anesthesia. We observed that the diameter of the left ovarian cyst was $5 \mathrm{~cm}$, the capsule was complete, and fat tissue was found on the surface, without adhesion to the surrounding tissues. The pathological examination showed that the cystic 
wall was locally thickened with a nodule of $1.0 \times 0.5 \mathrm{~cm}$. An intraoperative frozen section diagnosis confirmed a teratoma accompanied by a malignant transformation in the left ovary. Under general anesthesia, a laparoscopic total hysterectomy combined with an omentectomy and pelvic lymph node dissection was conducted. The postoperative pathological examination showed a teratoma with the malignant transformation of a sebaceous gland carcinoma in the left ovary accompanied by a lipogranuloma and normal uterus. There was no metastasis to the omentum majus or pelvic lymph nodes, and no tumor cells were found in the pelvic exfoliative cell examination.

The gross examination showed fat tissue in the cyst. The thickness of the cystic wall was $0.5-1.0 \mathrm{~cm}$, and a nodule of $1.0 \times 0.5 \mathrm{~cm}$ was found in the cystic wall. The nodule was light yellow with moderate hardness, and the remaining wall was off-white with moderate hardness. The microscopic examination showed mature teratoma components in the tumor tissue, including squamous epithelium, sebaceous glands, cartilage, and respiratory epithelium accompanied by a lipogranuloma. A sebaceous gland carcinoma component was observed in part of the tumor tissue. Immunohistochemically, the tumor cells were cytokeratin (CK) and androgen receptor (AR) positive, focally positive for CerBb2 and Ki67, and negative for the epithelial membrane antigen (EMA) (Figure 1).

The patient exhibited no abdominal pain, abdominal distension, lower limb pain, or other symptoms. Her preoperative diagnosis indicated a mature teratoma, and the B-mode ultrasound and CT scanning of the pelvic cavity did not detect any malignant transformation. However, the intraoperative frozen section diagnosis indicated a mature teratoma with a malignant transformation, while the pathological examination confirmed a sebaceous gland carcinoma. No metastatic lesions were found during the surgery. The pathological examination showed a normal uterus, with no metastasis to the omentum majus or pelvic lymph nodes. No tumor cells were found in the pelvic exfoliative cell examination. Therefore, this patient was diagnosed with a stage Ia mature teratoma according to the International Federation of Gynecology and Obstetrics (FIGO) staging.

\section{DISCUSSION}

Due to the different components of a teratoma, there can be various malignant transformations, such as squamous cell carcinomas (75\%-80\%), adenocarcinomas (7\%), sarcomas (7\%), basal cell carcinomas, sebaceous gland carcinomas, malignant melanomas, small cell lung cancers, transitional cell carcinomas, clear cell carcinomas, and gliomas ${ }^{[1,3-6]}$. The patholog- ical diagnosis confirmed that this case was a teratoma accompanied by the malignant transformation of a sebaceous gland carcinoma, which is very rare.

The mechanism of the malignant transformation of a mature teratoma remains unclear. Human papillomavirus (HPV) particles may retrograde to the ovaries via the ascending pathways of the cervix, uterus, and fallopian tubes, and the HPV capsid protein and HPV16/18E6 protein can be detected in patients with teratomas accompanied by malignant transformations of squamous cell carcinomas, while the detection rate is low in cystic teratomas. Therefore, a high risk HPV infection may play a role in inducing the transformation of the teratoma to a squamous cell carcinoma ${ }^{[3,7]}$. In this case, the malignant transformation of a sebaceous gland carcinoma negative for HPV was detected, but the possibility of a false negative is not negligible. Therefore, the role of HPV in the malignant transformation requires further exploration. Mature teratomas occur mainly in women of childbearing age (around 30 years old), whereas malignant changes are more common in menopausal women, which may be related to the presence of the teratoma and the long-term exposure to various malignant factors in the pelvic cavity ${ }^{[3]}$. Therefore, it is suggested that postmenopausal women undergo the active surgical treatment of ovarian tumors in order to prevent the malignant transformation caused by persistent ovarian tumors and the long-term exposure to malignant factors. Previous reports have shown that chromosomal aberrations, p53 gene mutations, and cyclooxygenase-2 play important roles in the malignant transformation of a teratoma. In addition, the low malignant transformation rate of a teratoma is related to the low expressions of p53 and p16 genes ${ }^{[6]}$. There are no special clinical symptoms in the early stage of a malignant transformation. However, the most common symptoms are a palpable abdominal mass, abdominal distension, and hypogastralgia, while the mild symptoms include lumbago, dysuria, weight loss, rupture, or torsion of the cyst. With a malignant transformation invading the surrounding organs or lymph nodes, clinical symptoms, such as a stomachache, leg pain, and lower extremity and vulvar edema, are aggravated ${ }^{[1,3,8]}$. In this case, the tumor in the left ovary was detected by B-mode ultrasound, and there were no clinical symptoms.

A mature teratoma can be diagnosed by ultrasonography, CT, or magnetic resonance imaging (MRI) scanning, but it is difficult to predict or diagnose the malignant transformation before surgery. A secondary teratoma with a malignant transformation is generally much larger in size, with $90 \%$ of these tumors falling between $10 \mathrm{~cm}$ and $20 \mathrm{~cm}$ in diameter. If the diameter is $\geq 10 \mathrm{~cm}$, the possibility of a malignant transforma- 
tion should be considered ${ }^{[1]}$. A B-mode ultrasound is the first choice for monitoring a teratoma ${ }^{[9]}$; therefore, when a B-mode ultrasound finds a giant pelvic mass, heterogeneous solid components in the cystic wall, cystic wall involvement, and adhesion of the tumor tissue to the surrounding tissues, the possibility of a malignant transformation should be highly considered. A mature teratoma has complex ultrasonic manifestations, and it has a common characteristic "lack of blood flow" signal. In a Doppler ultrasound, a high diastolic blood flow and low impedance can help to assess the presence of a malignant transformation ${ }^{[8,10]}$. An enhanced CT and MRI show higher resolution of the soft tissue and higher sensitivity to fat composition and calcification, but it is still difficult to predict the malignant transformation of a teratoma by CT and MRI scanning because of its complex tissue composition. When an enhanced CT or MRI shows a tumor diameter of $\geq 10$ $\mathrm{cm}$, cystic wall thickening, an unclear boundary, irregular lobulation, uneven solid component enhancement, transmural necrosis, surrounding tissue adhesion, and lymph node enlargement in the peritoneum, these symptoms indicate the possibility of invasive disease ${ }^{[5,11,12]}$. When compared with the traditional T1-weighted images, the fat-suppressed T1-weighted images show more clearly the contrast-enhanced solid part of the teratoma, which is helpful to predict the teratoma malignancy ${ }^{[7]}$. The possibility of a malignant transformation should be considered when a mature teratoma grows more than $2 \mathrm{~cm}$ per year ${ }^{[8]}$. Therefore, a preoperative B-mode ultrasound and pelvic enhanced CT or MRI examination are helpful for the early prediction of the malignant transformation of a teratoma. If a malignant transformation is suspected, a positron emission tomography-CT can be considered for the preoperative staging. In this case, the teratoma diameter was $5 \mathrm{~cm}$,

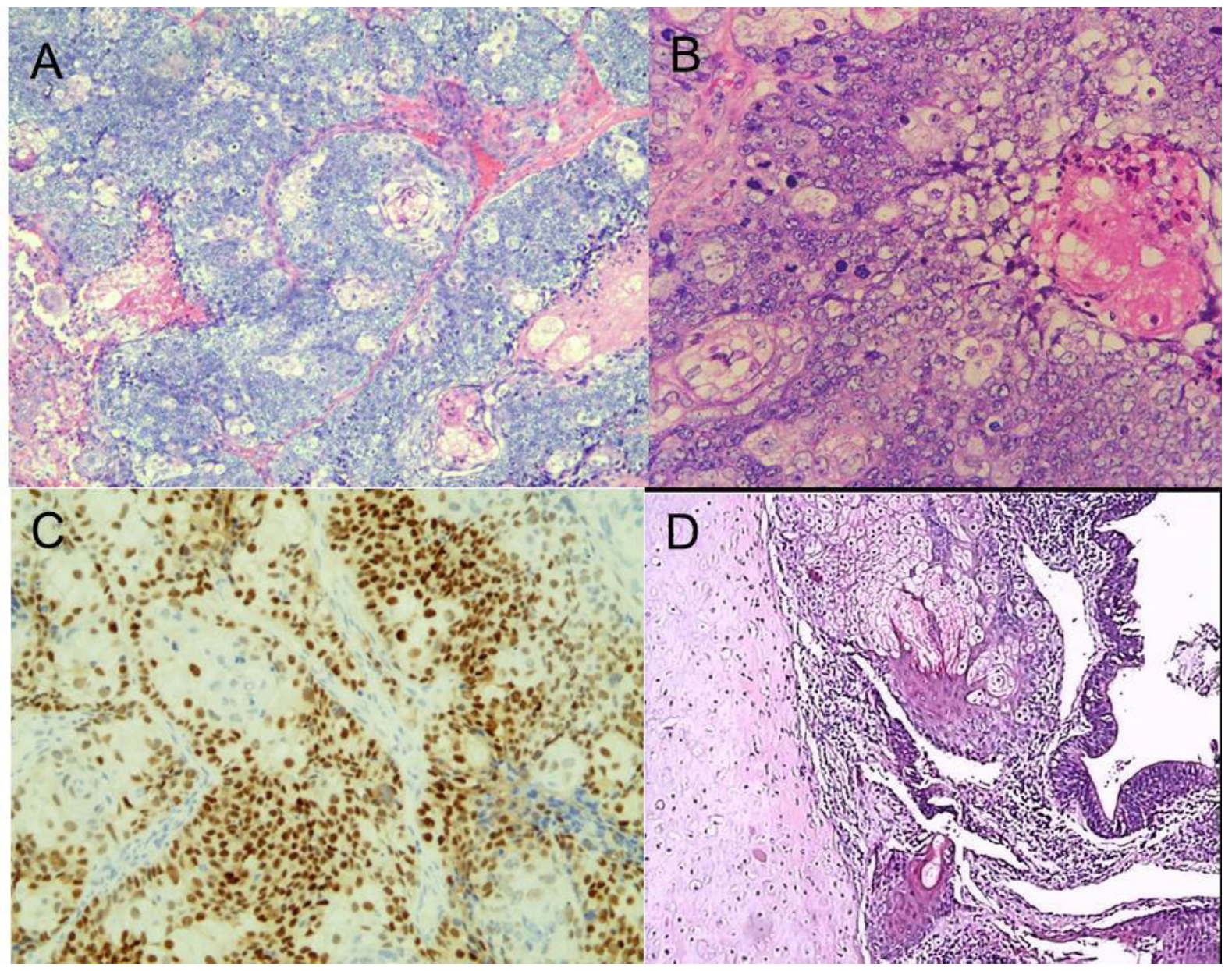

Figure 1. Pathological examination of a mature teratoma with a malignant transformation. (A) Lobulated structure with keratinization, a multinucleated giant cell reaction, and a necrosis "acne-like" structure. hematoxylin and eosin (HE) $\times 10$. (B) Microscopic examination showing basal cells and differentiated sebaceous gland cells. The nucleus of the tumor is large and vacuolar, with obvious eosinophilic nucleoli and nuclear division. HE $\times 40$. (C) Mature teratoma components, including squamous epithelium, sebaceous glands, cartilage, and metaplastic squamous epithelia. HE×10. (D) Immunohistochemistry showing tumor cells positive for the anti-androgen receptor. HE×20. 
and the B-mode ultrasound and CT scanning did not detect the malignant transformation of the teratoma, which might have been related to the complicated component of the teratoma.

In teratomas, the serum tumor markers, such as CA199, CA125, CEA, AFP, and SCC, should be examined before the operation. CA199 is closely related to mature teratomas, and SCC is the most relevant to mature teratomas with malignant transformations of squamous cell carcinomas. One retrospective study showed that the tumor marker concentration was independent of the FIGO staging. Thus, high CA125 levels may be associated with a poor prognosis. Currently, the diagnostic effects of the serum tumor markers on the malignant transformation of a teratoma are still controversial ${ }^{[3]}$. Researchers have also found that the CA125 and CA199 levels were closely related with the diameter of the teratoma and the prognosis of mature ovarian teratomas ${ }^{[13]}$. In this case, the serum CA199 level was upregulated, and the levels of the other tumor markers were within the normal range. However, the malignant transformation of the teratoma could not be determined by an elevated CA199 level.

The diagnosis of a mature ovarian teratoma depends on the surgical pathology. If there is a malignant transformation in the teratoma, fat and hair can be detected. There is also cystic wall thickening, a rough cystic wall, and adhesion to the surrounding tissues. Tumors show up as off-white or light yellow and brittle, and they are often accompanied by bleeding and necrosis. A malignant transformation often occurs in the inner papilla of the cyst wall, near the nodules, in the inhomogeneous enhanced portion or the thickening cyst wall ${ }^{[3,4,13]}$. Therefore, surgeons should carefully examine the cyst wall of the specimen. If nodules, papillae, and cyst wall thickening are found, there is a possibility of a malignant transformation. Surgeons should not blindly rely on the typical manifestations of mature ovarian teratomas, such as hair and teeth, which may lead to a misdiagnosis. Current studies have shown that the histological analysis of intraoperative frozen sections can identify teratomas with a malignant transformation, avoid secondary surgery to completely remove the tumor, and improve the life quality of these patients ${ }^{[3,4]}$.

Currently, the therapeutic selections for teratomas with malignant transformations remain controversial. However, most scholars have reached a consensus on surgical treatment. After an intraoperative frozen section diagnosis, a total hysterectomy combined with an omentectomy can be conducted. Because direct invasion and peritoneal metastasis are the main routes of tumor metastasis, it is still controversial to conduct pelvic and abdominal aortic lymph node dissections ${ }^{[3]}$.
For young and fertile patients in stage Ia, it is feasible to perform a unilateral adnexectomy combined with comprehensive staging surgery. Teratomas with malignant transformations are rare, so there is insufficient clinical data to support the safety of fertility-preserving surgery, even at early stages. Moreover, there is no consensus on the role of adjuvant chemotherapy in the treatment of teratomas with malignant transformations; however, the survival time of the patients who received adjuvant chemotherapy was longer than in those who did not ${ }^{[3]}$. Based on the effectiveness of alkylating agents and platinum drugs in the treatment of malignant ovarian epithelial tumors, researchers have suggested that adjuvant chemotherapy can be used for patients above stage Ib in order to improve the survival rate. Whole pelvic radiotherapy combined with cisplatin-based chemotherapy can benefit patients at stage Ic or stage II. However, some researchers doubt the effectiveness of pelvic radiotherapy ${ }^{[14]}$.

The prognosis is related to the FIGO stage, tumor growth pattern, histological type, histological differentiation, cyst rupture, vascular invasion, and presence of tumor cells in the ascites. Patients with tumor ruptures, vascular involvement, and specific pathological types (such as adenocarcinoma, sarcoma, etc.) have worse prognoses. One retrospective study showed that the early diagnosis and complete surgical resection of the tumor were important for improving the survival rate and avoiding potential metastasis ${ }^{[15]}$. Previous studies have shown that the 5-year survival rates of FIGO stage I, stage II, and stage III cases are 95\%, 80\%, and $0 \%$, respectively. Therefore, an early diagnosis of a malignant transformation before invasion or metastasis is particularly important for the prognosis. In this case, a yellow granular texture was found in the pelvic cavity during the surgery, and the possibility of metastasis was considered. Sebaceous gland carcinomas have a worse prognosis; therefore, under comprehensive consideration, a total hysterectomy combined with an omentectomy and pelvic lymph node dissection was conducted.

A malignant transformation can occur in any mature teratoma tissue ${ }^{[16]}$. In this case, the malignant transformation occurred in the sebaceous glands of the teratoma tissue. A sebaceous gland carcinoma is a rare skin malignancy, which derives from the sebaceous glands differentiated from the epidermal cells of the skin and appendages ${ }^{[17,18]}$. A sebaceous gland carcinoma can be divided into ocular and extraocular types. The former type accounts for $75 \%$ of sebaceous gland carcinomas, and these can be found in the eyelids and surrounding tissues ${ }^{[19,20]}$. The external ocular type occurs mainly in the head and neck with abundant sebaceous glands ${ }^{[21]}$. A sebaceous gland carcinoma malignantly trans- 
formed from the skin tissue of a teratoma is extremely rare. The occurrence and development of a sebaceous gland carcinoma is a long and complicated process, with multiple genes and steps, including the activation of proto-oncogenes, inactivation of tumor suppressor genes, and the abnormal expression of signal transduction molecules. Therefore, histochemistry and immunohistochemistry are meaningful in the diagnosis of sebaceous gland carcinomas. Lipids are enriched in tumor cells. After lipid dissolution, a negative reaction can be observed by glycogen staining in a paraffin section, and a positive reaction can be observed in oil red 0 staining and Sudan III staining for frozen section, which is beneficial for the diagnosis of a sebaceous gland carcinoma. Currently, there are no characteristic immunohistochemical markers for mature teratomas with malignant transformations, and the epithelial markers are all positive, such as CK, EMA, CD15, CU8, and CA15.3. In recent years, sebaceous gland carcinoma cells have been found to specifically express human lactoglobulin 2 (HMFGP-2) and AR proteins ${ }^{[22,23]}$. However, a sebaceous gland carcinoma has poor sensitivity to radiotherapy and chemotherapy. Thus, surgery is recommended as the main treatment, and its prognosis is related to the location, size, tumor stage, and differentiation degree of the tumor cells. Leaving no residual cancer cells at the cutting edge is the key to success. Moreover, the recurrence is positively correlated with the tumor size, so an early diagnosis is important for the prognosis of a sebaceous gland carcinoma ${ }^{[24,25]}$. Because there is limited research on the ocular type of sebaceous gland carcinoma, there is no specific diagnosis and treatment for a mature teratoma with a malignant transformation.

In conclusion, the early detection, early diagnosis, and early treatment of mature teratomas with malignant transformations are very important for improving the prognosis. For woman over 40 years old, when the image examination shows a tumor diameter $\geq 10 \mathrm{~cm}$, elevated serum tumor markers, cystic wall thickening, an unclear boundary, irregular lobulation, uneven solid component enhancement, transmural necrosis, adhesion to the surrounding tissue, and lymph node enlargement in the ascites and peritoneum, the possibility of the malignant transformation of a mature teratoma should be highly considered. Therefore, in order to improve the survival rate, it is important to pay attention to the preoperative risk assessment, optimize the preoperative and postoperative management, choose the best operative approaches, and emphasize the effectiveness of the intraoperative frozen assessment.

\section{REFERENCES}

1. Feng, X., and Xu, L. (2018) Rare case of squamous cell carcinoma arising in a recurrent ovarian mature cystic teratoma of a young woman: A case report and review of the literature. Medicine (Baltimore) 97, e10802

2. Oranratanaphan, S., and Khemapech, N. (2013) Characteristics and treatment outcomes of patients with malignant transformation arising from mature cystic teratoma of the ovary: experience at a single institution. Asian Pac. J. Cancer Prev. 14, 4693-4697

3. Chiang, A., Chen, M., Weng, C., Lin, H., Lu, C., Wang, P., Huang, Y., Chiang, Y., Yu, M., and Chang, C. (2017) Malignant transformation of ovarian mature cystic teratoma into squamous cell carcinoma: a Taiwanese Gynecologic Oncology Group (TGOG) study. J Gynecol Oncol 28, e69

4. Srivastava, P., Dawson, L., and Mandal, A. (2015) Squamous cell carcinoma arising in mature cystic teratoma with sigmoid invasion. J Cancer Res Ther 11, 1024

5. Chuang, H., Chen, Y., Mac, T., Chen, Y., Chen, H., Wang, W., and Tsai, E. (2015) Urothelial carcinoma arising from an ovarian mature cystic teratoma. Taiwan J Obstet Gynecol 54, 442-444

6. Zhu, H., Zou, Z., Lin, P., Li, W., Huang, Y., Shi, X., and Shen, H. (2015) Malignant transformation rate and p53, and p16 expression in teratomatous skin of ovarian mature cystic teratoma. Asian Pac. J. Cancer Prev. 16, 1165-1168

7. Takemori, M., and Nishimura, R. (2003) MRI findings of an ovarian dermoid cyst with malignant transformation. Magn Reson Med Sci 2, 105-108

8. Bonahy, A., Sabbah, H., Vadell, A., and Baba, N. (2017) [Malignant teratoma: about a case and a review of the literature]. Pan Afr Med J 27, 61

9. Werner, H., Mocarzel, C., Sá, R., Tonni, G., Novoa Y Novoa, V., Avvad-Portari, E., Bonasoni, P., and Araujo Júnior, E. (2016) Antenatal Diagnosis of a Large Immature Abdominal Wall Teratoma by 2D-3D Ultrasound Using HDlive and Magnetic Resonance Imaging. Fetal Pediatr Pathol 35, 434-441

10. Li, Y., Wen, G., and Yang, L. (2009) [Inspection on angiogenesis in malignant transformation of breast tumor by ultrasound contrast and quantitative analysis]. Zhonghua Yi Xue Za Zhi 89, 587-591

11. Kalampokas, E., Boutas, I., Kairi-Vasilatou, E., Salakos, N., Panoulis, K., Aravantinos, L., Damaskos, C., Kalampokas, T., and Deligeoroglou, E. (2014) A rare case report of squamous-cell carcinoma arising from mature cystic teratoma of ovary. G Chir 35, 241-245

12. Yarmohammadi, H., Mansoori, B., Wong, V., Tacher, V., Wilkins, L., Pavlidakey, P., and Haaga, J. (2014) Squamous cell carcinoma arising from ovarian mature cystic teratoma and causing small bowel obstruction. J Cancer Res Ther 10, 770-772

13. Tazo, Y., Yoshimura, Y., Shoda, T., Kyushima, N., Okada, T., and Yamazaki, H. (2016) Relevance of frozen sections and serum markers in invasive squamous cell carcinoma 
arising from ovarian mature cystic teratoma: two case reports. J Med Case Rep 10, 20

14. Kavitha, P., Priya, J. J., Katherine, K., Kevin, O. R., Matthew, Q. C., Nikhil, R., and Susana, C. (2011) Ovarian squamous cell carcinoma arising from mature cystic teratoma. Cancer Imaging 11, 67-69

15. Wen, K., Hu, W., Twu, N., Chen, P., and Wang, P. (2006) Poor prognosis of intraoperative rupture of mature cystic teratoma with malignant transformation. Taiwan J Obstet Gynecol 45, 253-256

16. Mathew, M., Ramdas, A., Kurian, S., Kuruvilla, L., and Singh, N. (2018) A Rare Malignant Transformation of an Ovarian Cystic Teratoma: A Case Report. Case Rep Pathol 2018, 6892783

17. Manteghi, A., Zwillenberg, S., and Arguello-Guerra, V. (2014) Sebaceous carcinoma of the parotid gland: a case report and review of the literature. Ear Nose Throat J 93, E29-32

18. Costea, C., Petraru, D., Dumitrescu, G., and Sava, A. (2013) Sebaceous carcinoma of the eyelid: anatomoclinical data. Rom J Morphol Embryol 54, 665-668

19. Shields, J., Saktanasate, J., Lally, S., Carrasco, J., and Shields, C. (2015) Sebaceous Carcinoma of the Ocular Region: The 2014 Professor Winifred Mao Lecture. Asia Pac J Ophthalmol (Phila) 4, 221-227

20. Jayaraj, P., Sen, S., Rangarajan, S., Ray, N., Vasu, K., Singh, V., Phartyal, R., Yadav, S., and Verma, A. (2018) Immunohistochemical evaluation of stress-responsive protein sestrin2 and its correlation with p53 mutational status in eyelid sebaceous gland carcinoma. Br J Ophthalmol $102,848-854$

21. Satomura, H., Ogata, D., Arai, E., and Tsuchida, T. (2017) Dermoscopic features of ocular and extraocular sebaceous carcinomas. J. Dermatol. 44, 1313-1316

22. Yunoki, T., Miyakoshi, A., Otsuka, M., and Hayashi, A. (2018) Clinicopathological features of considerable reduction in androgen receptor expression in sebaceous gland carcinoma of the eyelid. Int Ophthalmol

23. Mulay, K., Shah, S., Aggarwal, E., White, V., and Honavar, S. (2014) Periocular sebaceous gland carcinoma: do androgen receptor (NR3C4) and nuclear survivin (BIRC5) have a prognostic significance? Acta Ophthalmol 92, e681-687

24. Vianna, L., Cariello, A., Lowen, M., Sant'Anna, A., and Hofling-Lima, A. (2011) Sebaceous carcinoma of the eyelid - different diagnostic times, different outcomes: case reports. Arq Bras Oftalmol 74, 444-446

25. Hou, J., Killian, J., Baum, C., Otley, C., Roenigk, R., Arpey, C., Weaver, A., and Brewer, J. (2014) Characteristics of sebaceous carcinoma and early outcomes of treatment using Mohs micrographic surgery versus wide local excision: an update of the Mayo Clinic experience over the past 2 decades. Dermatol Surg 40, 241-246Yin, J., Wang, X., Wang, C., Huang, J., Zhang, C., Chen, L., Wang, C., Geng, X., and Ma, X. (2016) Selection of internal fixation for posterior malleolar fractures. Chinese Journal of Orthopaedic Trauma 18, 545-549 